\title{
Continuous Healthcare
}

\section{Removing the Inconvenience of Recharging}

\author{
Patrick Bentsen, Jesper Hedeager Nielsen, \\ Jørgen Nybo, Christen Ring, Stefan Wagner \\ Electronics Department \\ Aarhus School of Engineering \\ Aarhus, Denmark
}

\begin{abstract}
The OpenCare Personal Communication Device (OCPCD) is an extension of the OpenCare Service Engine infrastructure in the shape of a mobile wrist or neck-born device for monitoring users vital signs and activity-level while not at home, and for allowing the user to remote control his home and communicate with others using the build-in cell phone module. It also provides fall detection by integrating with an external fall detection module. The biggest challenge for mobile devices such as the OCPCD is the power usage level in combination with limited battery resources. The OCPCD has therefore experimentally been equipped with ubiquitous wireless recharging features, assuring that the user will not have to remove the device or connect it with any wires while recharging.
\end{abstract}

Keywords- wireless charging; wireless power; pervasive healthcare; mobile device

\section{INTRODUCTION}

For continuous and pervasive healthcare monitoring in the users home, many research teams have advocated for the use of a mobile device, e.g. a cell phone, as the primary or even sole platform for sensor integration and communication purposes $[1,2,3]$. This approach has various flaws however, including the limited processing power of the device, the limited bandwith and number of communication channels (for instance limiting Bluetooth communication to one sensor at a time, not allowing for ZigBee and proprietary protocols etc). The most significant shortcoming of the mobile device approach however, is the limited battery resources. Measurements indicate that it is not possible to maintain continuous healthcare monitoring of a cell phone based device for prolonged periods of time, due to limited battery power. Depending on the mobile device and attached sensor types, often less than 24 hours of battery life is achieved. This is of course not in any way acceptable for non-autonomous users, who require help to perform the recharging functions, as the battery needs to be charged or changed at an unacceptable frequency requiring external help.

As an alternative, it is possible to use a stationary platform as a sensor gateway with a wall plug-in power supply. This solves the power limitations but comes at a cost: less mobility for the user, and thus limiting monitoring functions to the user's home.

We have developed a platform for pervasive healthcare monitoring in the users own home [4], where we combine a stationary plugged power device with a mobile device, the OCPCD. This OCPCD mobile device is not meant in the role of a separate gateway device, but instead interacts with a stationary computer unit, the Basestation, mounted in the apartment of the user. This device samples and distributes data from all sensor types, including for instance fall detectors, ECG, blood-pressure, weight, automatic medicine dispenser units $[4,5]$ and others. The OCPCD is only assuming the role as a communication link and gateway for the sensors when the user has left his home (and is not within reach of the stationary basesation unit). Thus it will not use battery power while the user is at home.

Even though this means less strain on the battery power of the OCPCD device while at home, it still does not allow for prolonged careteake-intervention-free usage. Recharing is still needed on a regular baisis. In this paper we will demonstrate a prototype which recharges with no need for a wired connection, while the user is sitting at the table or lying in bed.

\section{SYSTEM DESCRIPTION}

\section{A. OCPCD Device}

The system is designed as a mobile device to be worn around the wrist (or potentially around the neck). It simplifies everyday life for the user by making basic tasks easier to accomplish and handles potential sensor communication.

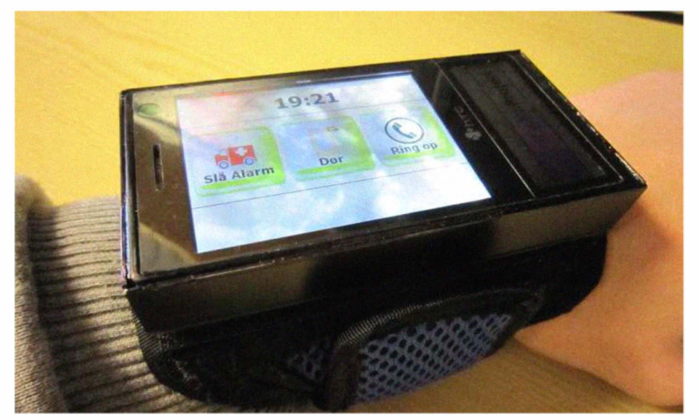

Figure 1. The OCPCD device showing a part of the user interface, including the alert button, the "open door" button, and the phone button.

The OCPCD is thus a device designed to never have to leave the wearers body, or demand intervention from caretakers, 
which requires it to be able to recharge without user intervention. This is done using wireless power recharging.

\section{B. Wireless charging setup}

To charge wirelessly we use the principle of Coupled Magnetic Resonance. [6]

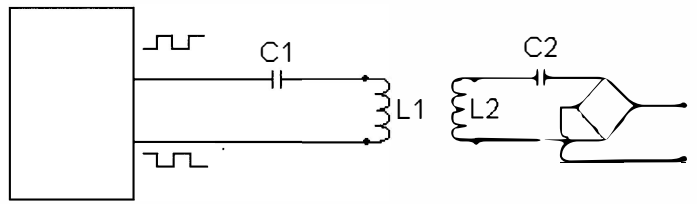

Figure 2. The Source coil (L1) is connected to an oscillating circuit, switching the direction of the current through the coil with a $50 \%$ duty-cycle The induced voltage in the device coil (L2) is rectified and connected to the $3.6 \mathrm{~V}$ rechargeable battery in the OCPCD, and charges the OCPCD in this way.

The Source and the device circuits are both tuned to resonate at the same frequency. Due to practical circumstances L1(D1 $=30 \mathrm{~cm})$ and $\mathrm{L} 2(\mathrm{D} 2=3 \mathrm{~cm})$ are of different size. The coupling between L1 and L2 is loose. Only approximately $1 \%$ of the magnetic flux of the source inductor (L1) is couple to the device inductor (L2). Therefore the device inductor is L2 = $100^{*} \mathrm{~L} 1$. In this way the induce voltage in the device is increased 10 times.

The system is designed to work at $100 \mathrm{kHz}$ and the components $\mathrm{C} 1$ and $\mathrm{C} 2$ are chosen accordingly.

With the OCPCD located at the center of the source coil the charging current reaches $300 \mathrm{~mA}$ and the efficiency is $11 \%$.

The OCPCD may be recharged at a distance of up 30 centimeters, allowing the source coil to be mounted under a table or within the mattress of the user's bed for nighttime recharging.

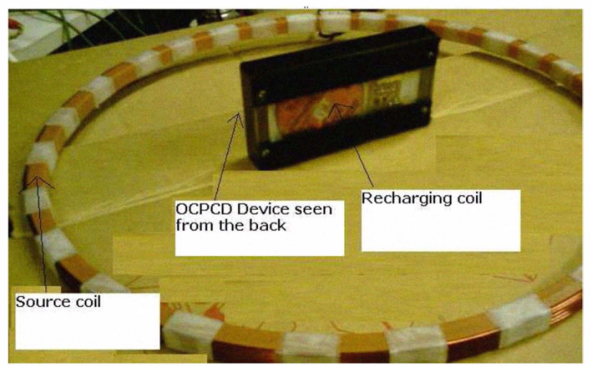

Figure 3. The two coils in the prototype. The source coil transmitting the power, and the reciever coil embedded in the OCPCD (seen from the back)

\section{DEMONSTRATIONS}

We will demonstrate that the OCPCD can be charged using wireless power, and the general use of the worn device. We will also discuss alternative usages, including charging other healthcare devices.

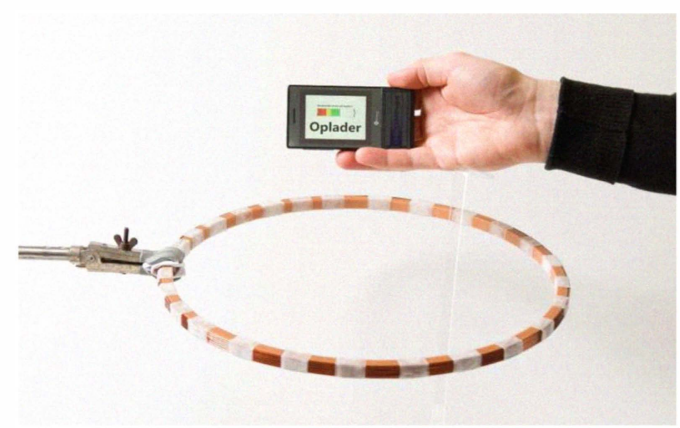

Figure 4. An image of the recharging OCPCD in a distance of $30 \mathrm{~cm}$. from the source coil..

\section{REFERENCES}

[1] H.J. Lee, S.H. Lee, K. Ha, H.C. Jang, W. Chung, J.Y. Kim, et al., "Ubiquitous healthcare service using Zigbee and mobile phone for elderly patients". International Journal of Medical Informatics 2009 3;78(3):193-198

[2] R. Lee, C. Hsiao, C. Chen, M. Liu, "A Mobile-Care System Integrated with Bluetooth Blood Pressure and Pulse Monitor, and Cellular Phone". IEICE Trans.Inf.Syst. 2006 /5/1;E89-D(5):1702-1711

[3] A.Lorenz, R. Oppermann, "Mobile health monitoring for the elderly: Designing for diversity", Pervasive and Mobile Computing, Volume 5 , Issue 5, October 2009, Pages 478-495,

[4] S. Wagner, C. Nielsen, "OpenCare Project: An Open, Flexible and Easily Extendible Infrastructure for Pervasive Healthcare Assisted Living Solutions". Pervasive Computing Technologies for Healthcare, 2009. 3rd International Conference on, 2009

[5] S. Wagner, "Zero-configuration of pervasive healthcare sensor networks" Proceedings of the The Third International Conference on Pervasive Computing and Applications (ICPCA2008), Alexandria, Egypt, 2008.

[6] Wireless Power Transfer via Strongly Coupled Magnetic Resonances Originally published in Science Express on 7 June 2007, Science 6 July 2007:Vol. 317. no. 5834, pp. $83-86$ 